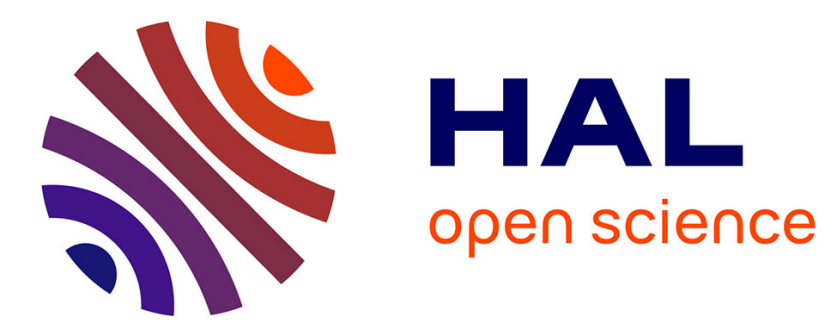

\title{
Commande optimale à flux libre des machines asynchrones
}

\author{
J. Biedinger, S. Poullain, J. Yvon
}

\section{To cite this version:}

J. Biedinger, S. Poullain, J. Yvon. Commande optimale à flux libre des machines asynchrones. Journal de Physique III, 1992, 2 (1), pp.57-78. 10.1051/jp3:1992256 . jpa-00248727

\section{HAL Id: jpa-00248727 https://hal.science/jpa-00248727}

Submitted on 1 Jan 1992

HAL is a multi-disciplinary open access archive for the deposit and dissemination of scientific research documents, whether they are published or not. The documents may come from teaching and research institutions in France or abroad, or from public or private research centers.
L'archive ouverte pluridisciplinaire HAL, est destinée au dépôt et à la diffusion de documents scientifiques de niveau recherche, publiés ou non, émanant des établissements d'enseignement et de recherche français ou étrangers, des laboratoires publics ou privés. 
Classification

Physics Abstracts

41.10

\title{
Commande optimale à flux libre des machines asynchrones
}

\author{
J. M. Biedinger, S. Poullain et J. P. Yvon \\ Université de Technologie de Compiègne, BP 649, 60206 Compiègne Cedex, France
}

(Reçu le 28 février 1991, révisé le 8 juillet 1991, accepté le 20 septembre 1991)

\begin{abstract}
Résumé. - Dans cet article on traite le problème du contrôle optimal à flux libre d'un moteur asynchrone considéré comme un système à paramètres répartis gouverné par une équation de diffusion non linéaire. Cette approche permet de décrire l'état électrodynamique de la machine en prenant naturellement en compte le effets de la saturation et des courants induits, ainsi que les non-linéarités liées au fonctionnement à flux variable. La formulation éléments finis est utilisée pour traduire le problème continu sous la forme d'un système dynamique de dimension finie pour lequel les techniques du contrôle optimal peuvent s'appliquer. La généralité de la méthodologie permet de traiter différents critères d'optimisation. Ici l'objectif est de maîtriser l'évolution du couple électromagnétique, en particulier lorsque le moteur est initialement démagnétisé, avec naturellement des contraintes sur les courants; à cet effet le critère est défini comme une norme de la différence entre le couple électromagnétique et une consigne de couple. Les calculs sont effectués pour un moteur asynchrone à cage et un moteur à rotor massif. Les résultats sont comparés avec ceux déduits de stratégies basées sur des modèles à constantes localisées. Les réponses obtenues à partir de commandes vectorielles adaptées pour tenir compte du fonctionnement à flux variable sont également présentées. On indique finalement comment modifier le critère pour traiter le problème de la recherche de la capacité maximale de réponse en couple des moteurs.
\end{abstract}

\begin{abstract}
The subject of this paper is the optimal control with unconstrained flux of an induction motor being considered as a distributed parameter system described by a nonlinear diffusion equation. This approach allows to describe the electrodynamic state of the motor by taking into account, in a natural way, the influence of saturation and eddy-currents, as well as the nonlinearities due to the variable flux operation. Equations are approximated via a finite element formulation which leads to a finite dimensional dynamical system to which the techniques of optimal control may be applied. The generality of the methodology allows to treat various criteria. The objective aimed in this paper is to force the motor torque to follow a given input, especially under initially demagnetized conditions and with, naturally, constraints on the currents. For this purpose the criterion will be chosen as a norm of the difference between the electromagnetic torque and a given reference torque. Numerical simulations are presented for the case of a squirrel-cage rotor and for a solid iron rotor. Results are compared with those deduced from strategies based on lumped-parameter models. Torque responses obtained with fieldoriented control method adapted to the case of flux-variable operation are also presented. Finally a modification of the criterion is proposed for identifying the command which allows to obtain the larger torque during transient states.
\end{abstract}




\section{Introduction.}

Pour répondre à des exigences dynamiques élevées, les actionneurs modernes sont commandés suivant la technique du contrôle vectoriel. Celle-ci représente en fait la synthèse d'une loi de contrôle optimal en couple dont la valeur peut être modifiée instantanément conformément à sa consigne [1, 2].

Mais une telle synthèse repose sur deux hypothèses fondamentales. La première admet la possibilité d'identifier l'état électrodynamique de la machine à partir d'un modèle linéaire à constantes localisées, ce qui donne accès à l'amplitude $\psi_{\mathrm{r}}$ et à la phase (direction d) du flux rotorique. La seconde suppose le flux rotorique $\psi_{\mathrm{r}}$ préalablement établi à la valeur souhaitée ; en maintenant ce flux constant, il est possible d'annihiler les régimes transitoires magnétiques qui pénaliseraient la rapidité de réponse en couple. Ces hypothèses permettent de définir une commande qui assure théoriquement un fonctionnement sans régime transitoire: la consigne des courants statoriques est élaborée en assignant une valeur constante à leur composante $i_{\text {sd }}$ en phase avec le flux $\psi_{\mathrm{r}}$, et en pilotant le couple moteur $c_{\mathrm{cm}}$ par la composante $i_{\mathrm{sq}}$ en quadrature $\left(c_{\mathrm{em}}=K i_{\mathrm{sq}} \psi_{\mathrm{r}}\right)$.

Alors que la première hypothèse peut être partiellement relaxée en adaptant le modèle linéaire de base pour tenir compte des non-linéarités [3], la seconde suppose des conditions magnétiques incompatibles avec les fonctionnements à flux variable. Ce sont par exemple les fonctionnements où les phases de repos prédominent devant les phases de travail. Dans ces conditions, piloter le moteur avec une commande à flux constant serait trop pénalisant pour le rendement (exemple du moteur asynchrone utilisé comme actionneur de direction assistée de véhicule automobile [4]). Une stratégie de commande à flux nul pendant les phases de repos est alors préférable. Mais en contrepartie la vitesse d'accroissement du couple est ralentie au début des phases de travail, car la commande doit assurer en plus l'établissement du flux $\psi_{r}$, lequel ne peut subir de variations rapides à cause d'une constante de temps élevée. Dans de telles conditions, on conçoit qu'une commande vectorielle élaborant la consigne de couple sur la base d'un flux rotorique constant, ne constitue plus une méthode optimale pour gérer l'évolution du couple suivant une trajectoire imposée. Un des problèmes est de savoir dans quelles limites une telle loi de commande n'est plus optimale.

L'objectif de cet article est de présenter une méthodologie générale d'identification des lois de contrôle optimal en couple des machines électriques, en particulier dans le cas de stratégies à flux libre. La solution de ce problème repose sur les techniques du contrôle optimal. Mais une condition essentielle à leur mise en œuvre réside dans l'utilisation d'un modèle mathématique suffisamment complexe permettant de prédire le comportement du système avec précision. Or, dans le cas d'une commande à flux libre, deux phénomènes rendent difficile l'identification d'un modèle à constantes localisées : la saturation magnétique et l'effet pelliculaire. On peut toujours adapter les modèles à constantes localisées pour tenir compte de ces non-linéarités. Mais outre leurs limites à réaliser un tel objectif (voir par exemple [5] dans le cas d'une commande vectorielle à flux imposé), ces modèles se prêtent difficilement à un traitement analytique des techniques du contrôle optimal dès que l'on introduit plusieurs variables de commande [6].

Pour s'affranchir des limitatons inhérentes aux modèles à constantes localisées, nous considérons le problème de la commande optimale en couple d'un moteur comme celui du contrôle optimal d'un système gouverné par des équations aux dérivées partielles [7]. Les phénomènes de saturation et d'effet pelliculaire sont ainsi explicitement incorporés dans le modèle estimant l'état électrodynamique du moteur. La formulation éléments finis permet de réduire le problème continu à un système dynamique de dimension finie auquel on peut appliquer les techniques du contrôle optimal. Un élément esentiel à la mise en œuvre de ces 
techniques consiste à calculer le gradient du critère d'optimisation par rapport à la commande. La méthode utilisée repose sur la résolutio' de l'équation adjointe [8].

Mais la définition du critère d'optimisation n'est pas unique. Elle est en fait imposée par le type de performances souhaitées pour le moteur. Pcur illustrer la méthodologie, c'est le problème du suivi de trajectoire en couple qui a été retenu : le critère consiste en une norme de l'écart entre le couple électromagnétique et une consigne de couple, avec naturellement des contraintes sur les courants de commande.

Après avoir rappelé la théorie sous la forme du problème continu, nous donnons les étapes de l'algorithme de résolution du problème discrétisé par éléments finis. Nous discutons ensuite de la prise en compte des contraintes sur les courants de commande. L'application porte sur la recherche de la réponse optimale en couple, au sens du problème de suivi de trajectoire, pour un moteur asynchrone initialement démagnétisé. Pour juger de l'influence du modèle, nous reportons également les réponses optimales élaborées à partir de modèles à constantes localisées, dans le cas d'un rotor à cage, puis d'un moteur à rotor en fer massif. En développant cette méthodologie, notre objectif premier était de fournir une référence aux méthodes plus conventionnelles de commande des machines électriques. Aussi nous reportons également des résultats de simulations de commandes vectorielles adaptées pour tenir compte de la situation à flux variable. Nous proposons à cet effet une stratégie de commande vectorielle simple pour approcher l'optimum.

L'objectif de réaliser une trajectoire en couple peut se révéler inadapté dans certaines circonstances, par exemple pour les applications où c'est l'obtention du maximum de couple qui est souhaitable. Mais la méthodologie présentée est suffisamment générale pour pouvoir s'adapter à des critères définis en ce sens. Ceci est illustré au travers un critère qui assure un maximum de couple en régime transitoire à flux variable, et qui permet ainsi de caractériser la capacité limite de réponse en couple d'un moteur, compte tenu bien sûr des contraintes sur les courants.

Soulignons enfin qu'avec l'objectif de la maîtrise du couple, la vitesse mécanique apparaît comme une grandeur secondaire (la production du couple repose essentiellement sur la vitesse relative du rotor par rapport au champ magnétique inducteur); on la supposera constante pour simplifier la formulation éléments finis. Le problème plus général du contrôle optimal de la vitesse en temps minimal ne sera pas abordé ici (notons toutefois que la résolution d'un tel problème conduit obligatoirement à maximiser le couple électromagnétique; en ce sens les résultats présentés constituent les prémisses d'une étude plus générale).

\section{Le problème d'optimisation.}

Pour définir le problème d'optimisation, il faut préciser l'équation d'état, la commande et le critère. L'approche utilisée consiste à décrire l'état électrodynamique de la machine par les potentiels du champ électromagnétique dont l'évolution est régie par les équations de Maxwell dans l'approximation des états quasi-stationnaires. Avec l'hypothèse d'invariance suivant la direction $\mathbf{u}_{x_{3}}$, et en supposant le milieu conducteur du rotor simplement connexe, cet état est entièrement décrit par l'unique composante $A\left(x_{1}, x_{2}, t\right)$ du potentiel vecteur magnétique $\mathbf{A}$ :

$$
\mathbf{A}(\mathbf{x}, t)=A\left(x_{1}, x_{2}, t\right) \mathbf{u}_{x_{3}}, \quad \mathbf{B}=\operatorname{rot} \mathbf{A},
$$

qui est solution de l'équation de diffusion non linéaire

$$
\left\{\begin{array}{l}
\gamma \frac{\partial A}{\partial t}-\mathrm{D}(A)+\gamma\left(V_{x_{1}} \frac{\partial A}{\partial x_{1}}+V_{x_{2}} \frac{\partial A}{\partial x_{2}}\right)=J_{\mathrm{s}}(\mathbf{u}) \text { sur } \Omega, \\
A(\mathbf{x}, t)=0 \text { sur } \Gamma(\Omega), \\
A\left(\mathbf{x}, t_{0}\right)=A_{0}(\mathbf{x}), \text { conditions initiales sur } \Omega .
\end{array}\right.
$$

JOURNAL, DE PHYSIQUE III $-\Upsilon 2, N^{*}$ I, JANVIER 1992 
Dans cette équation $\mathrm{D}$ représente l'opérateur elliptique

$$
\mathrm{D}(A)=\sum_{i=1}^{2} \frac{\partial}{\partial x_{i}}\left(\frac{1}{\mu(B)} \frac{\partial A}{\partial x_{t}}\right),
$$

$\gamma$ la conductivité électrique, $V_{x_{1}}$ et $V_{x_{2}}$ les composantes du champ de vitesse du milieu conducteur supposé constant dans le temps, et $\mu(B)$ la perméabilité magnétique.

La fonction densité de courant $J_{s}(\mathbf{u})$ traduit la répartition des $q$ courants d'alimentation $\mathbf{i}_{\text {s }}$ dont le contrôle dépend des paramètres de commande notés $\mathbf{u}(t)$. Le potentiel vecteur $A$ défini par (2) est fonction de $\mathbf{u}$; la notation $A(\mathbf{x}, t ; \mathbf{u})$ souligne cette dépendance fonctionnelle. Le nombre de degrés de liberté attachés aux courants $\mathbf{i}_{\mathrm{s}}$ détermine la dimension $m$ du vecteur commande $\mathbf{u}$. En fonction des contraintes, qu'il reste à préciser, l'espace $\mathrm{U}_{\mathrm{ad}}$ des commandes admissibles se définit formellement comme

$$
\mathrm{U}_{\mathrm{ad}}=\left\{\mathbf{u} \mid \mathbf{u}(t) \in K \subset \mathbb{R}^{m}\right\} .
$$

On désire minimiser l'écart entre le couple instantané $c_{\mathrm{em}}(t)$ délivré par le moteur et sa consigne $c^{*}(t)$ sur l'intervalle de temps $\left[t_{0}, t_{\mathrm{f}}\right]$. Le critère $J(\mathbf{u})$ est défini par

$$
J(\mathbf{u})=\int_{t_{0}}^{t_{\mathrm{f}}}\left\{\int_{\Omega} \Phi[A(\mathbf{x}, t ; \mathbf{u}), \mathbf{x}, t] \mathrm{d} \Omega\right\} \mathrm{d} t \equiv \int_{t_{0}}^{t_{\mathrm{f}}}\left|c_{\mathrm{em}}(t ; \mathbf{u})-c^{*}(t)\right|^{2} \mathrm{~d} t .
$$

L'intégration du tenseur de contraintes de Maxwell sur un contour fermé $\Sigma$ entourant le rotor permet de relier le couple à l'état magnétique par

$$
c_{\mathrm{em}}(t ; u)=\int_{\Sigma} \tau[A(s, t ; \mathbf{u})] \mathrm{d} s .
$$

Avec ces données, le problème d'optimisation en couple se résume ainsi : parmi les commandes admissibles $\mathbf{u}(t) \in \mathrm{U}_{\mathrm{ad}}$ qui transfèrent le système de l'état initial imposé $A\left(\mathrm{x}, t_{0}\right)$ à l'état final libre $A\left(\mathrm{x}, t_{\mathrm{f}}\right)$ suivant l'équation d'évolution (2), trouver la commande optimale admissible $\tilde{\mathbf{u}}(t)$ qui minimise le critère (5).

La solution à ce problème théorique est fournie par l'extension de la théorie de la commande optimale au cas de systèmes gouvernés par des équations aux dérivées partielles [7]. Celle-ci montre que la forme de l'opérateur $\mathrm{D}(A)$ assure l'existence d'un contrôle optimal $\tilde{\mathbf{u}}$. La condition nécessaire de l'optimalité de $\tilde{\mathbf{u}}$ s'exprime par l'existence d'une fonction $\lambda(t)$ vérifiant le système différentiel

$$
\left\{\begin{array}{l}
\gamma \frac{\partial \lambda}{\partial t}+\mathrm{D}^{*}(A)+\gamma \sum_{i=1}^{2} \frac{\partial}{\partial x_{i}}\left(\lambda V_{x_{l}}\right)=-\frac{\partial \Phi(A, \mathbf{x}, t)}{\partial A} \text { sur } \Omega \\
\lambda(\mathbf{x}, t)=0 \text { sur } \Gamma(\Omega) \\
\lambda\left(\mathbf{x}, t_{\mathrm{f}}\right)=0 \text { sur } \Omega .
\end{array}\right.
$$

où $\mathrm{D}^{*}$ l'opérateur adjoint de $\mathrm{D}$ (ici $\mathrm{D}^{*} \equiv \mathrm{D}$ si $\mu$ est indépendant de $B$ ). dans ces conditions, il est démontré dans [7] que le contrôle optimal ũ est caractérisé par (2), (7) et

$$
\nabla_{\tilde{u}} J \cdot(\mathbf{u}-\tilde{\mathbf{u}})=\int_{t_{0}}^{t_{\tilde{f}}}\left\{\int_{\Omega}\left\{\lambda(t) \frac{\partial J_{\mathrm{s}}(\mathbf{x}, t ; \mathbf{u})}{\partial \mathbf{u}}\right\}_{\tilde{\mathbf{u}}} \mathrm{d} \Omega\right\}\{\mathbf{u}(t)-\tilde{\mathbf{u}}(t)\} \mathrm{d} t \geqslant 0 . \quad \forall \mathbf{u}(t) \in \mathrm{U}_{\mathrm{ad}} .
$$

Etant donnée la nature non linéaire du problème, le système d'équations (2), (7) ne peut être résolu directement. Il est donc nécessaire d'utiliser une méthode d'optimisation itérative 
qui requiert, en général, le calcul du gradient de $J$ par (8). La détermination de ce dernier passe par la résolution de l'équation adjointe (7), ce qui nécessite une résolution numérique de (2), (7). On développe à cet effet le calcul par une formulation éléments finis. Il est alors intéressant de noter, d'après (2) et (7), que les conditions aux limites sur $\Gamma(\Omega)$ sont de même type pour les fonctions $A$ et $\lambda$; c'est-à-dire que leur formulation éléments finis conduira à manipuler des matrices de même topologie, ce qui facilitera l'implantation de l'algorithme numérique.

Pour plus de généralité, on notera par la suite $X$ l'état électrodynamique du système qui, avec l'hypothèse de la vitesse constante, se réduit ici au potentiel vecteur : $X(\mathbf{x}, t ; \mathbf{u}) \equiv A(\mathbf{x}, t ; \mathbf{u})$.

\section{Approximation éléments finis.}

La technique des éléments finis appliquée à une formulation variationnelle de type Galerkine de l'équation (2) conduit à rechercher une approximation du potentiel $A$ sous la forme

$$
A\left(x_{1}, x_{2}, t\right)=\sum_{i=1}^{n} N_{1}\left(x_{1}, x_{2}\right) A_{1}(t)
$$

et à modéliser la machine par un système dynamique de dimension finie $n$ :

$$
\left\{\begin{array}{l}
\mathrm{M} \frac{\mathrm{dX}(t)}{\mathrm{d} t}+\mathrm{K}(\mathrm{X}(t)) \mathrm{X}(t)=\mathbf{d}(\mathbf{u}(t)) \\
X\left(t_{0}\right)=\mathrm{X}_{0}
\end{array}\right.
$$

où

- $\mathrm{X}(t) \in \mathbb{R}^{n}$, représente les valeur nodales du potentiel $A\left(M_{j}\right)_{j}=1 ., n$,

- $n$ est le nombre de valeurs nodales libres (les conditions aux limites sur $A$ et $\lambda$ étant identiques, on peut les incorporer formellement dans l'écriture matricielle),

- $\mathrm{M}$ et $\mathrm{K}$ sont des matrices $(n \times n)$ définies comme

$$
\begin{gathered}
\mathrm{M}: M_{\imath \jmath}=\int_{\Omega} \gamma N_{\imath} N, \mathrm{~d} \Omega, \\
\mathrm{K}(\mathrm{X}): K_{\imath \jmath}=\int_{\Omega}\left\{\frac{1}{\mu(\mathrm{X})} \nabla N_{\imath} \nabla N_{j}+\gamma N_{i} \sum_{k=1}^{2} V_{x_{k}} \frac{\partial N_{\jmath}}{\partial_{x_{k}}}\right\} \mathrm{d} \Omega,
\end{gathered}
$$

- $\mathbf{d}(\mathbf{u})=\mathrm{Ci}_{\mathrm{s}}(\mathbf{u})$ est un vecteur $(n \times 1)$, la matrice $\mathrm{C}$ étant définie par

$$
\begin{gathered}
C_{1 j}=\frac{n s_{J}}{S_{J}} \int_{\Omega} N_{1} \mathrm{~d} \Omega: \text { nœud } i \in \text { bobine (section } S_{J}, n s_{J} \text { spires) } \\
=0 \begin{aligned}
\quad \text { nœud } i \notin \text { bobine } \mathrm{j} \\
i=1 . n, j=1 . q .
\end{aligned}
\end{gathered}
$$

Le critère relatif au suivi de trajectoire fait intervenir le couple moteur, lequel est calculé au moyen du tenseur de Maxwell comme une fonction quadratique de l'état:

$$
c_{\mathrm{em}}(t)=\mathrm{X}(t)^{\top} \mathrm{QX}(t)
$$

où $Q$ est une matrice constante, indépendante de l'état de saturation, qui ne dépend que des caractéristiques géométriques de l'intégrale curviligne de (6) située dans l'entrefer. 
Le problème consiste à trouver la commande admissible $\tilde{\mathbf{u}}(t)$ telle que

$$
J(\tilde{\mathbf{u}}) \leqslant J(\mathbf{u})=\int_{t_{0}}^{t_{f}}\left|\left(\mathrm{X}(t)^{\top} \mathrm{QX}(t)-c^{*}(t)\right)\right|^{2} \mathrm{~d} t \quad \forall \mathbf{u} \in \mathrm{U}_{\mathrm{ad}} .
$$

Des conditions nécessaires pour un contrôle optimal ont été données par (2), (7) et (8). Leur transposition dans le cadre d'une formulation éléments finis des équations peut être dérivée du calcul standard des variations [8]. La fonction de Lagrange

$$
L=J(\mathbf{u})+\int_{t_{0}}^{t_{\mathrm{f}}} \lambda(t)^{\top}\left\{\mathrm{d}(\mathbf{u})-\mathrm{K}(\mathrm{X}) \mathrm{X}-\mathrm{M} \frac{\mathrm{dX}}{\mathrm{d} t}\right\} \mathrm{d} t
$$

doit être stationnaire pour toute variation admissible de la commande optimale le long d'une trajectoire admissible $X(t)$. Après intégration par partie, $L$ se met sous la forme

$$
\begin{aligned}
L=\int_{t_{0}}^{t_{\mathrm{f}}}\left|\mathrm{X}(t)^{\top} \mathrm{QX}(t)-c^{*}(t)\right|^{2} \mathrm{~d} t & +\int_{t_{0}}^{t_{\mathrm{f}}} \lambda(t)^{\top}\{\mathbf{d}(\mathbf{u})-\mathrm{K}(\mathrm{X}) \mathrm{X}\} \mathrm{d} t+ \\
& +\int_{t_{0}}^{t_{\mathrm{f}}}\left(\mathrm{M}^{\top} \frac{\mathrm{d} \lambda}{\mathrm{d} t}\right)^{\top} \mathrm{X} \mathrm{d} t-\left[\mathrm{M}^{\top} \lambda\right]_{t_{\mathrm{f}}}^{\top} \mathrm{X}\left(t_{\mathrm{f}}\right),
\end{aligned}
$$

ce qui conduit à exprimer sa variation première $\delta L$ comme

$$
\begin{aligned}
\delta L= & \int_{t_{0}}^{t_{\mathrm{f}}} 2\left\{\mathrm{X}^{\top} \mathrm{QX}-c^{*}(t)\right\}(\overline{\mathrm{Q} X})^{\top} \delta \mathrm{X} \mathrm{d} t+ \\
& +\int_{t_{0}}^{t_{\mathrm{f}}}\left\{\left(\mathrm{M}^{\top} \frac{\mathrm{d} \lambda}{\mathrm{d} t}\right)^{\top}-\left(\mathrm{K}(\mathrm{X})^{\top} \lambda\right)^{\top}-\left(\mathrm{KT}(\mathrm{X})^{\top} \lambda\right)^{\top}\right\} \delta \mathrm{X} \mathrm{d} t+ \\
& +\int_{t_{0}}^{t_{\mathrm{f}}}\left(\mathrm{D}(\mathbf{u})^{\top} \lambda\right)^{\top} \delta \mathbf{u} \mathrm{d} t-\left(\mathrm{M}^{\top} \lambda\left(t_{\mathrm{f}}\right)\right)^{\top} \delta \mathrm{X}\left(t_{\mathrm{f}}\right),
\end{aligned}
$$

avec :

$$
\begin{gathered}
\overline{\mathrm{Q}}=\mathrm{Q}+\mathrm{Q}^{\top}, \\
\mathrm{D}(\mathbf{u}): D_{j k}=\frac{\partial d_{j}(\mathbf{u})}{\partial u_{k}}, \quad j=1 . n, \quad k=1 . m,
\end{gathered}
$$

$\mathrm{KT}(\mathrm{X}): K T_{1 j}=\sum_{k=1}^{n} \sum_{\ell=1}^{n} 2 X_{k} X_{\ell} \times$

$$
\times \int_{\Omega}\left[\frac{\partial \nu}{\partial B^{2}(X)}\left\{\nabla N_{\imath} \cdot \nabla N_{k}\right\}\left\{\nabla N_{l} \cdot \nabla N_{\ell}\right\}\right] \mathrm{d} \Omega, i, j=1 . n .
$$

(KT s'identifie à la matrice jacobienne qui intervient lors de la résolution de (2) par la méthode de Newton-Raphson).

Pour annuler $\delta L$, l'état adjoint $\lambda(\mathbf{x}, t)$ doit être solution de l'équation

$$
\left\{\begin{array}{l}
-\mathrm{M}^{\top} \frac{\mathrm{d} \lambda}{\mathrm{d} t}+(\mathrm{K}(\mathrm{X})+\mathrm{KT}(\mathrm{X}))^{\top} \lambda=2\left(\mathrm{X}^{\top} \mathrm{QX}-c^{*}(t)\right) \overline{\mathrm{Q}} \mathrm{X}, \\
\lambda\left(t_{\mathrm{f}}\right)=0
\end{array}\right.
$$


Dans ces conditions le contrôle optimal ũ est caractérisé par (10), (22) et

$$
\nabla_{\tilde{\mathbf{u}}} J \tilde{\mathbf{u}} \cdot \delta \tilde{\mathbf{u}} \equiv \nabla_{\tilde{\mathbf{u}}} L \cdot \delta \tilde{\mathbf{u}}=\int_{t_{0}}^{t_{\mathrm{f}}}\left(\mathrm{D}^{\top} \lambda\right)^{\top} \delta \tilde{\mathbf{u}}(t) \mathrm{d} t \equiv 0
$$

\section{Solution du problème de contrôle optimal.}

L'algorithme de recherche du contrôle optimal ũ procède par itération sur les commandes en vue d'annuler le gradient $\nabla_{u} J$, ce qui représente la condition nécessaire de stationnarité (15). Une paramétrisation adaptée de la commande permet de ramener ce problème à un problème de programmation mathématique. Cette paramétrisation peut être prise comme

$$
\mathbf{u}(t)=\sum_{k=1}^{s} \mathbf{v}_{k} w_{k}(t), \quad \mathbf{v}_{k} \in \mathbb{R}^{m}
$$

où les fonctions $\left(w_{k}\right)_{k=1} \ldots s$ sont données (par exemple constantes par morceau ou linéaires). La détermination de $\tilde{\mathbf{u}}$ sur $\left[t_{0}, t_{\mathrm{f}}\right]$ est alors équivalente à celle de $s$ vecteurs $\widetilde{\mathbf{v}}_{\mathrm{k}}$. Comme le calcul du gradient par (23) nécessite l'intégration numérique de (10) et (22) avec une discrétisation temporelle de l'intervalle d'étude $\left[t_{0}, t_{\mathrm{f}}\right]$ en $N$ intervalles $\Delta T$, une paramétrisation possible pourra être choisie comme

$$
\mathrm{U}_{\mathrm{ad}}=\left\{\mathbf{u} \mid \mathbf{u}(t)=\mathbf{v}_{k}, \quad k \Delta T \leqslant t<(k+1) \Delta T, k=0 . . N-1\right\} .
$$

Ainsi le critère (15) devient une fonction algébrique $J(\mathbf{v})$ des $m N$ composantes du vecteur $\mathbf{v}$ défini comme

$$
\mathbf{v}=\left\{\mathbf{v}_{0}, \mathbf{v}_{1}, \ldots, \mathbf{v}_{N-1}\right\}^{\top} \in \mathbb{R}^{m N}
$$

et la condition nécessaire d'optimalité (23) prend la forme

$$
\nabla_{v k} J=\mathrm{D}\left(\mathbf{v}_{k}\right)^{\top} \lambda_{k}, \quad k=0 . . N-1 .
$$

L'intégration des équations (10), (22) est réalisée avec un schéma d'Euler implicite :

$$
\left\{\begin{array}{l}
\mathrm{M} \frac{\mathrm{X}_{k+1}-\mathrm{X}_{k}}{\Delta T}+\mathrm{K}\left(\mathrm{X}_{k+1}\right) \mathrm{X}_{k+1}=\mathrm{d}\left(\mathrm{v}_{k}\right), k=0 . . N-1, \\
\mathrm{X}_{0}=\text { condition initiale, }
\end{array}\right.
$$

$$
\left\{\begin{array}{l}
-\mathrm{M}^{\top} \frac{\lambda_{k}-\lambda_{k-1}}{\Delta T}+\left(\mathrm{K}\left(\mathrm{X}_{k}\right)+\mathrm{KT}\left(\mathrm{X}_{k}\right)\right)^{\top} \lambda_{k-1}= \\
=2\left(\mathrm{X}_{k}^{\top} \mathrm{QX}_{k}-c^{*}(k \Delta T)\right) \overline{\mathrm{Q}} \mathrm{X}_{k}, \quad k=N .1, \\
\lambda_{N}=0 .
\end{array}\right.
$$

Compte tenu de ces résultats, chaque pas de la procédure itérative pour déterminer $\tilde{\mathbf{v}}$ comportera les étapes suivantes :

a) intégrer (28) pour la commande $\mathbf{v}$, et stocker $\mathrm{X}_{k}, k=1 . N$;

b) intégrer (29) à rebours et stocker $\lambda_{k}, k=N-1.0$;

c) calculer le gradient (27) du critère par rapport aux $m N$ paramètres d'optimisation $\mathbf{v}$;

d) corriger, en accord avec les contraintes, l'estimation $\mathbf{v}$ de la commande optimale selon la méthode de quasi Newton implantée dans le module OPTIM du code Basile [9]. 


\section{Contraintes sur la commande.}

La définition des $m$ fonctions de commande $\mathbf{u}$, et donc des $N m$ composantes de $\mathbf{v}$ pour la version discrétisée, ainsi que le traitement des contraintes associées, dépend du type de source de courant utilisée. On examine deux possibilités : une source délivrant $q$ courants indépendants, et une source délivrant un système $q$-phasé équilibré de courants.

5.1 Source DÉlivrant $q$ COURANTS INDÉPENDANTS. - Dans le cas général d'une alimentation délivrant $q$ courants indépendants, les contraintes consistent à limiter l'amplitude maximale de chaque courant :

$$
\left|i_{\ell}\right| \leq I_{\mathrm{Max}} ; \quad \ell=1 . q,
$$

et à assurer que la somme instantanée de ces courants reste nulle:

$$
\sum_{\ell=1}^{4} i_{\ell} \equiv 0
$$

Or le module OPTIM utilisé ne supporte que des contraintes de bornes du type (30). Ces contraintes présentent l'avantage de pouvoir être traitées par projection, mais elles sont incompatibles avec les contraintes (31). Il faut procéder comme suit : si, à chaque intervalle $k \Delta T$ de discrétisation de $\left[t_{0}, t_{\mathrm{f}}\right]$, on exprime le q-ième courant de phase en fonction des $q-1$ autres courants :

$$
i_{q, h}=-\sum_{\ell=1}^{\prime} i_{1, k}, \quad k=0 \ldots N-1 \text {, }
$$

on peut identifier les $m$ composantes de la commande discrétisée $\mathbf{v}_{k}$ aux $q-1$ premiers courants de phase :

$$
\mathbf{v}_{h}=\left\{i_{1, k}, i_{2, k}, \ldots, i_{q-1, k}\right\}, \quad k=0 \ldots N-1,
$$

et écrire le second membre de (28) comme :

$$
\mathbf{d}\left(\mathbf{v}_{k}\right)=\mathrm{C}^{\prime} \mathbf{v}_{k}, \quad k=0 . . N-1,
$$

avec

$$
C^{\prime}=C \times\left[\begin{array}{rrr}
1 & 0 & 0 \\
0 & 1 & 0 \\
. & . & 1 \\
0 & 0 & -1
\end{array}\right] \text {, }
$$

sous les contraintes

$$
\begin{gathered}
\left|v_{\ell, k}\right| \leqslant I_{\text {Max }}, \quad \ell=1 . \quad m, \quad k=0 . . N-1 \\
\left|i_{q, k}\right| \equiv\left|\sum_{\ell=1}^{m} v_{\ell, k}\right| \leqslant I_{\operatorname{Max}}, \quad k=0 . . N-1 .
\end{gathered}
$$

Sous cette forme, les contraintes (35) peuvent être traitées directement par projection avec le module OPTIM. La contrainte (36) peut être introduite en pénalisant le critère (15) comme

$$
J(\mathrm{v})=\sum_{k=1}^{N}\left|\mathrm{X}_{k}^{\top} \mathrm{QX}_{k}\right|^{2}+\rho \sum_{k=0}^{N-1}\left\{\left(\left[I_{\mathrm{Max}}-i_{q k}\right]^{-}\right)^{2}+\left(\left[I_{\mathrm{Max}}+i_{q, k}\right]^{-}\right)^{2}\right\} \text {. }
$$


Dans cette expression la fonction $[\theta]^{-}$est définie comme

$$
[\theta]^{-}=\operatorname{Min}(\theta, 0) \text {. }
$$

Le facteur de pénalisation $\rho$ ne dépend pas de l'état $X$. Il n'a aucune incidence sur le calcul de l'état adjoint $\lambda$, mais il modifie le calcul de $\nabla_{\checkmark} J$ : par rapport à la paramétrisation $\mathbf{v}_{k}$ de la commande au pas de temps $k \Delta T$, ce gradient s'exprime comme

$$
\nabla_{\mathrm{v}_{k}} J=\mathrm{C}^{\prime \top} \lambda_{k}+2 \rho\left(\left[I_{\mathrm{Max}}-i_{q, k}\right]^{-}-\left[I_{\mathrm{Max}}+i_{q, k}\right]^{-}\right) \mu
$$

où $\mu$ représente un vecteur unitaire ayant ses $m$ composantes égales à 1 .

La difficulté consiste à choisir correctement la valeur de $\rho$ : trop élevée, elle pénalise la réalisation du couple ; trop faible, elle relaxe trop la contrainte (36). Le cas suivant est plus facile à mettre en œuvre.

5.2 SoURCE DÉliVRANT UN SYSTÈME q-PHASÉ ÉQuILIBRÉ DE COURANTS. - Dans la pratique les alimentations électroniques sont asservies à délivrer des systèmes $q$-phasés équilibrés de courants sinusoïdaux de la forme

$$
i_{\ell}(t)=I_{\mathrm{M}}(t) \times \cos \left(\psi(t)-\frac{2(\ell-1) \pi}{q}\right), \quad \ell=1 . \quad q .
$$

La commande discrétisée est ainsi restreinte aux $2 m$ degrés de liberté

$$
\mathbf{v}=\left\{\left(I_{\mathrm{M}, k}, \psi_{k}\right)_{k=0 \ldots N-1}\right\}
$$

qui vérifient implicitement la contrainte (31). Dans ces conditions le module OPTIM permet de prendre en compte toutes les contraintes sans pénaliser le critère. Le vecteur second membre $\mathbf{d}\left(\mathbf{v}_{k}\right)$ de $(28)$ devient :

$$
\begin{aligned}
& \mathbf{d}\left(\mathbf{v}_{k}\right)= \\
& =\mathrm{C}\left\{\mathbf{v}_{1, k} \cos \left(\mathbf{v}_{2, k}\right), \mathbf{v}_{1, k} \cos \left(\mathbf{v}_{2, k}-\frac{2 \pi}{q}\right) \cdot, \mathbf{v}_{1, k} \cos \left(\mathbf{v}_{2, k}-\frac{(m-1) 2 \pi}{q}\right)\right\}^{\top},
\end{aligned}
$$

ce qui permet d'évaluer l'expression (20) de D intervenant dans le gradient $\nabla_{\mathrm{v}} J(23)$. Les simulations numériques présentées ci-dessous procèdent de cette approche.

\section{Application.}

La méthodologie est appliquée pour étudier la réponse en couple d'un moteur asynchrone initialement démagnétisé et soumis à un échelon de consigne de couple. L'étude porte sur le moteur considéré suivant trois configurations (ses caractéristiques sont données en annexe 1) :

- avec un rotor à cage peu profonde sans saturation magnétique (configuration MCL);

- avec un rotor à cage peu profonde en tenant compte de la saturation magnétique (configuration MCNL);

- avec un rotor en acier massif (configuration MARM).

On compare les réponses en couple obtenues à partir de diverses stratégies de contrôle :

- le contrôle optimal à flux libre élaboré à partir du modèle éléments finis (contrôle référencé C.O.E.F.) ;

- le contrôle optimal à flux libre élaboré à partir du modèle à constantes localisées (contrôle référencé C.O.S.E., avec l'extension C.O.S.E.L. dans le cas linéaire, et 
C.O.S.E.N.L. dans le cas saturé) ; l'annexe 2 donne l'équivalent des équations de l'élat (10) et de l'adjoint (22) adaptées au modèle à constantes localisées ;

- une commande vectorielle élaborée à partir du modèle à constantes localisées, et dimensionnée pour créer en priorité le flux rotorique : les courants statoriques sont pilotés initialement avec une composante $i_{\mathrm{sq}}$ nulle tant que le flux rotorique n'a pas atteint la valeur minimale $\psi_{\mathrm{r}_{\mathrm{m} i n}}$ nécessaire pour réaliser la consigne de couple, compte tenu de la contrainte $I_{\text {Max }}$ sur l'amplitude; la consigne des courants est ensuite modifiée avec une composante $i_{\text {sd }}$ juste nécessaire pour maintenir $\psi_{\mathrm{r}}=\psi_{\mathrm{r}_{m n}}$, et une composante $i_{\mathrm{sq}}=\sqrt{I_{\text {Max }}^{2}-i_{\mathrm{sd}}^{2}}$ assurant une valeur de couple $c_{\mathrm{cm}}=\mathrm{K} \psi_{\mathrm{r}_{\mathrm{m}}} i_{\mathrm{sq}}$ égale à sa consigne (commande référencée C.V.F.M.) ;

- une commande vectorielle élaborée dans des conditions similaires à la précédente, mais en n'autorisant une composante de couple $i_{\text {sq }}$ non nulle que lorsque le flux rotorique a atteint la valeur qui conduirait en régime stationnaire au maximum de couple par ampère absorbé [10] (commande référencée C.V.C./I $I_{\text {Max }}$ );

- la commande imposant le courant statorique à la valeur réalisant la consigne de couple en régime stationnaire (commande référencée C.C.I.) ; la simulation du régime transitoire est réalisée sur le modèle éléments finis.

6.1 INFLUENCE DE LA PARAMÉTRISATION SUR LA COMMANDE OPTIMALE. - Le nombre $m N$ de paramètres d'optimisation (26) dépend de la finesse $N$ de la discrétisation temporelle du schéma d'intégration numérique des équations. L'objectif est de cerner la discrétisation minimale à partir de laquelle la commande optimale n'évolue plus de manière significative. On applique à et effet la méthodologie C.O.E.F. sur la configuration MCL, pour une consigne de couple de $5 \mathrm{Nm}$, et une amplitude maximale des courants de 2,25 A efficace, le moteur étant initialement démagnétisé. La figure 1 reporte les réponses optimales en couple obtenues pour des pas de discrétisation s'échelonnant de 1 à $20 \mathrm{~ms}$. Compte tenu de la valeur de la constante de temps rotorique $\tau_{\mathrm{r}}\left(\tau_{\mathrm{r}} \simeq 0,1 \mathrm{~s}\right)$, on peut estimer qu'une discrétisation temporelle avec un pas de temps $\Delta T=\frac{\tau_{\mathrm{r}}}{20}$ constitue un bon compromis entre la recherche de la meilleure réponse optimale et les temps calculs nécessaires. Ces derniers varient beaucoup avec le choix de la commande initiale, des contraintes sur les courants, et bien sûr

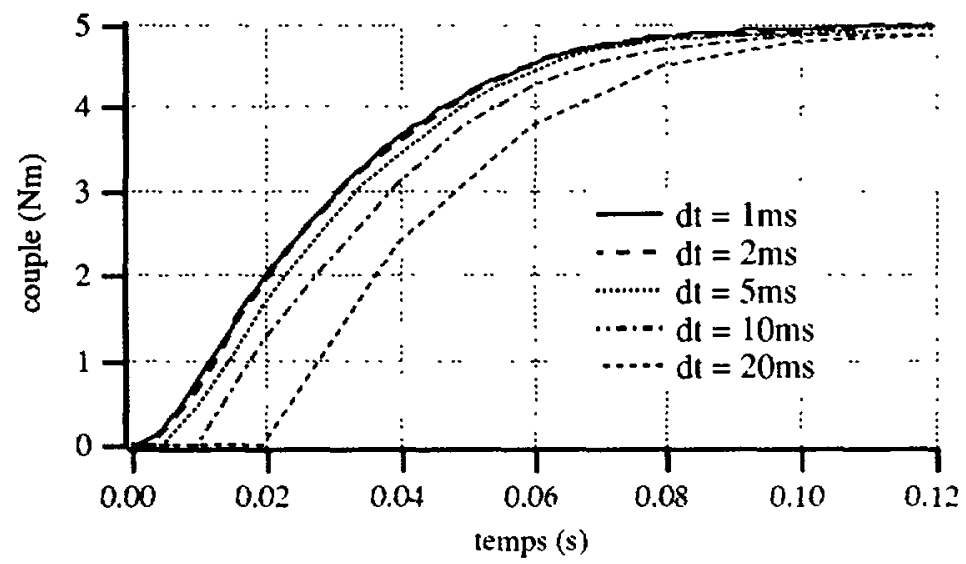

Fig. 1. - Stratégie C.O.E.F. : influence de la paramétrisation sur la commande optimale.

[C.O.E.F. method : influence of parametrization on the optimal control.] 
l'ordinateur. A titre indicatif, les temps CPU pour une itération sur la commande (étapes a, b, $\mathrm{c}, \mathrm{d}$ du §4) se répartissent ainsi : quand il faut 1 unité de temps pour le calcul d'optimisation par la méthode de quasi Newton avec Basile, celui de l'adjoint (22) en nécessite 1000 , et celui de l'état (10), 10 000. Avec une station de travail Apollo 4 500, les exemples traités dans cet article ont nécessité de 30 à 120 min par commande, le nombre de commandes traitées variant de 10 à 30. Pour la suite de l'étude les pas de discrétisation seront choisis comme $\Delta T=\frac{\tau_{\mathrm{r}}}{20}$.

6.2 CONFIGURATION MCL : ÉTUdE DE LA RÉPONSE EN COUPLE. - On recherche dans quelles mesures le moteur est capable de réaliser instantanément son couple nominal à partir de l'état démagnétisé. La figure 2 donne les réponses en couple obtenues avec les stratégies de contrôle C.O.E.F., C.O.S.E.L., C.V.F.M., C.V.C./I ${ }_{\mathrm{Max}}$, et C.C.I., pour la configuration $\mathrm{MCL}$, ainsi que les différents critères réalisés. La consigne de couple est de $5 \mathrm{Nm}$, et le courant admissible de 2,25 A efficace. D'après les résultats de l'annexe 1, la stratégie de commande C.C.I. doit imposer un courant de phase

$$
i(t)=2,25 \sqrt{2} \cos (2 \pi \times 0,253 \times t)
$$

pour donner le couple de $5 \mathrm{Nm}$ en régime stationnaire.

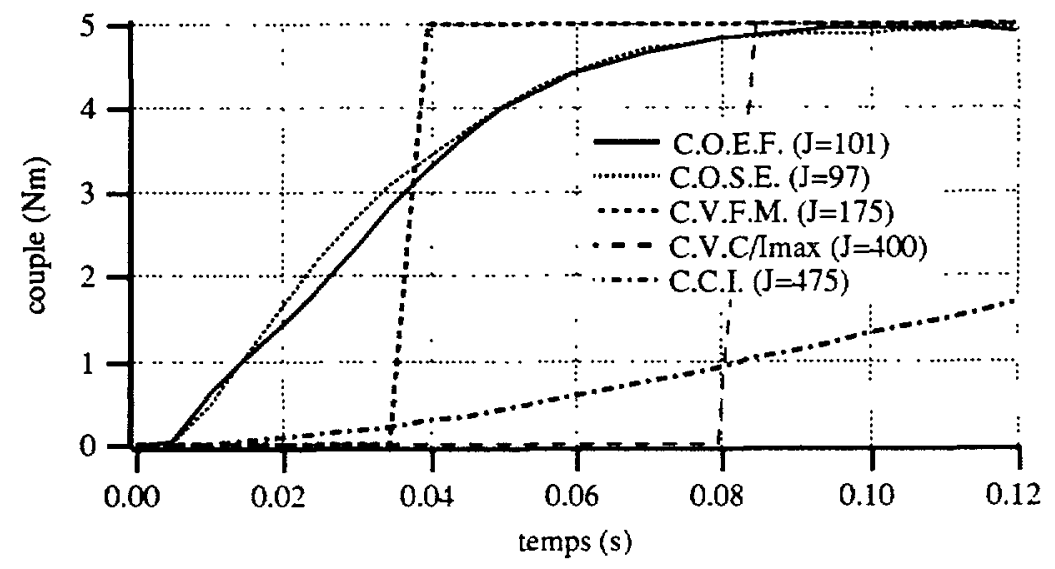

Fig. 2. - Modèle MCL : réponses en couple $c^{*}=5 \mathrm{Nm}-I_{\mathrm{Max}}=2,25 \mathrm{~A}$ eff).

[MCL model : torque responses $\left(c^{*}=5 \mathrm{Nm}-I_{\mathrm{Max}}=2.25 \mathrm{~A}\right.$ eff $\left.).\right]$

On constate que les modélisations par éléments finis et par schéma équivalent conduisent pratiquement à la même réponse optimale (les deux modèles étant linéaires, et la cage peu profonde, les paramètres du schéma équivalent évoluent peu avec les conditions de fonctionnement). Au vue de la valeur du critère, la stratégie de commande vectorielle C.V.F.M. réalise un bon compromis simplicité-précision. Par contre la stratégie de commande vectorielle C.V.C./I $I_{\mathrm{Max}}$, qui privilégie en fait le rendement [10], se révèle moins adaptée pour répondre à un problème de suivi de trajectoire. Remarquons également la médiocre réponse réalisée avec la stratégie C.C.I.

Les modèles à constantes localisées nécessitent plus d'hypothèses pour leur définition. Afin de juger de leur aptitude réelle à modéliser correctement l'état magnétique de la structure 
pour élaborer la commande, on effectue les simulations suivantes : les trajectoires optimales de courant élaborées avec ces modèles servent à simuler le comportement transitoire du moteur à l'aide d'un calcul de champ par éléments finis. La figure 3 reporte les réponses en couple correspondantes. Dans le cas de figure du moteur non saturé, ces résultats confirment, comme cela était prévisible, la validité du modèle à constantes localisées. La même démarche sera reprise pour les différents cas de figures étudiés par la suite.

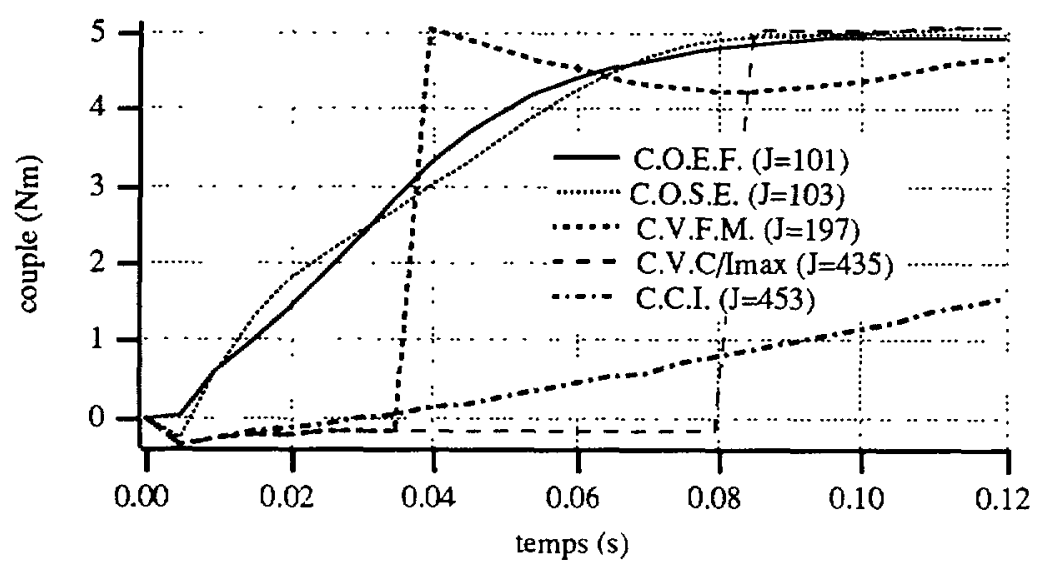

Fig. 3. - Modèle MCL : simulation par éléments finis des réponses en couple associées aux trajectoires de courant déduites des modèles à constantes localisées ( ${ }^{*}=5 \mathrm{Nm}-I_{\mathrm{Max}}=2,25 \mathrm{~A}$ eff $)$.

[MCL model : torque responses with transient finite element field computation corresponding to current policies determined by optimal control with lumped-parameter model $\left(c^{*}=5 \mathrm{Nm}-I_{\max }=\right.$ 2.25 A eff).]

6.3 CONFIGURATION MCNL : INFLUENCE DE LA SATURATION. - On examine l'influence de la saturation sur la réponse optimale en couple à partir de la configuration saturable MCNL (paramètres de l'annexe 1 pour le modèle à constantes localisées). L'objectif est le même que précédemment : réaliser le plus rapidement possible un échelon de consigne de couple de $5 \mathrm{Nm}$ en limitant le courant à 2,25 $\mathrm{A}$ efficace. La figure 4 donne les réponses en couple obtenues avec les stratégies de contrôle C.O.E.F., C.V.F.M. et C.C.I. Pour juger de l'influence de la saturation magnétique, la stratégie de contrôle optimal avec modèle à constantes localisées est présentée pour la configuration linéaire $\mathrm{MCL}$ du moteur (stratégie C.O.S.E.L.) ainsi que pour la configuration non linéaire MCNL (stratégie C.O.S.E.N.L.). D'après les caractéristiques de l'annexe 1 , il faut maintenant imposer une fréquence de $2,8 \mathrm{~Hz}$ pour obtenir en régime stationnaire un couple de $5 \mathrm{Nm}$ avec un courant de 2,25 A efficace.

Dans ce cas de figure, les réponses optimales en couple déduites des modèles à constantes localisées et du modèle éléments finis se différencient peu, car la saturation intervient seulement en fin de trajectoire. Mais si on utilise la séquence de courants des stratégies C.O.S.E.L. et C.O.S.E.N.L. pour une simulation éléments finis du régime transitoire associé, on constate que les modèles à constantes localisées commencent à perdre de leur validité. C'est ce qu'indique la figure 5. La même détérioration intervient pour la stratégie de commande C.V.F.M. La stratégie C.C.I. apparaît par contre améliorée en regard du cas précédent: elle est basée en effet sur un courant correspondant à un fonctionnement stationnaire saturé, alors qu'au démarrage le flux est encore nul, ce qui, compte tenu de la fréquence de glissement plus élevée, accélère la croissance du flux. Mais dans tous les cas, la 


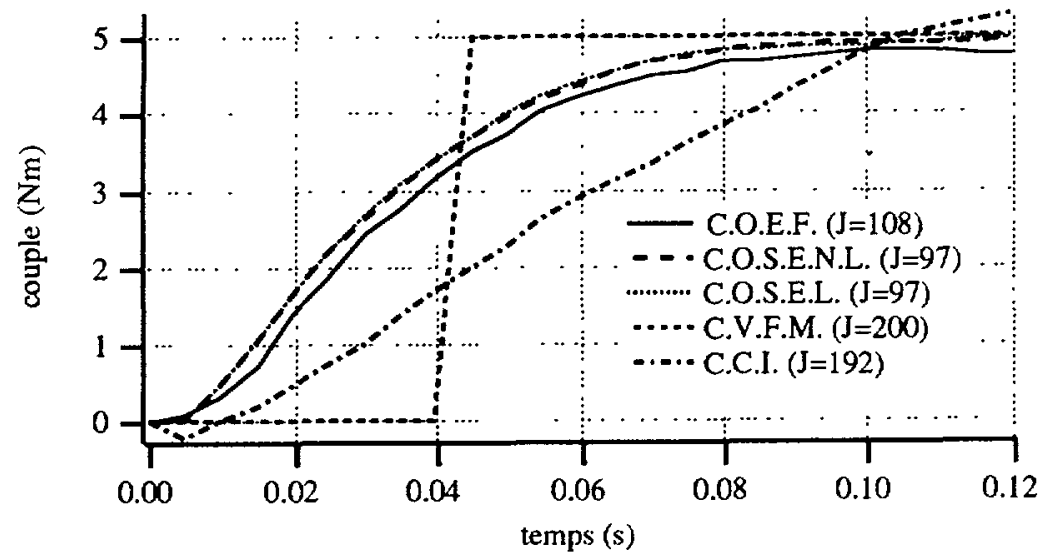

Fig. 4. - Modèle MCNL : réponses en couple $\left(c^{*}=5 \mathrm{Nm}-1_{\text {Max }}=2,25 \mathrm{~A}\right.$ eff $)$.

[MCNL model : torque responses $\left(c^{*}=5 \mathrm{Nm}-I_{\mathrm{Max}}=2.25 \mathrm{~A}\right.$ eff $)$.]

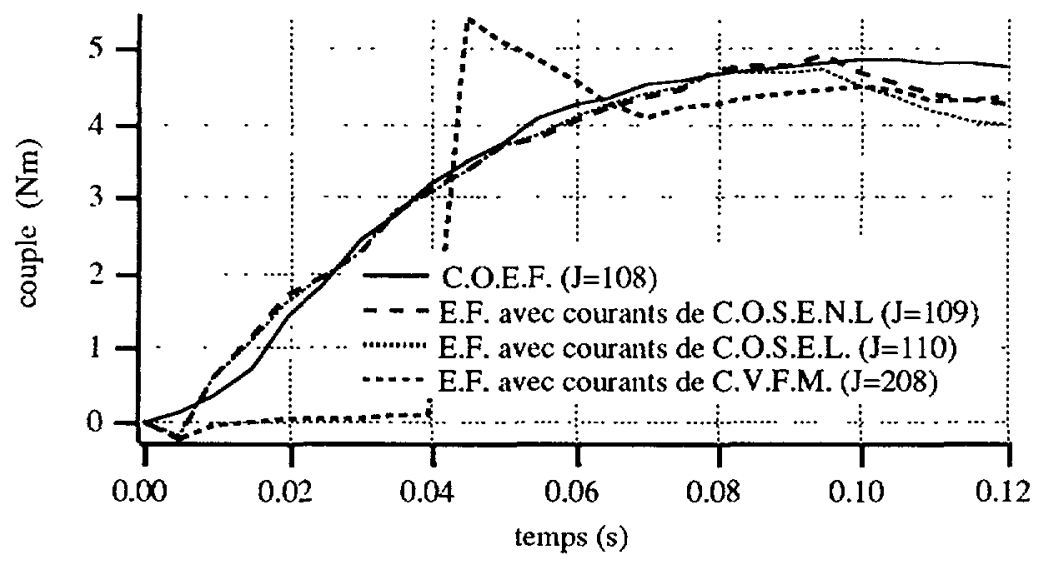

Fig. 5. - Modèle MCNL : simulation par éléments finis des réponses en couple réelles associées aux trajectoires de courant des modèles à constantes localisées $\left(c^{*}=5 \mathrm{Nm}-I_{\mathrm{Max}}=2,25 \mathrm{~A}\right.$ eff).

[MNCL model : torque responses with transient finite element field computation corresponding to current policies determined by optimal control with lumped-parameter model $\left(c^{*}=5 N m-I_{\text {Max }}=2.25\right.$ A eff $)$.]

méthodologie du contrôle optimal avec éléments finis conduit bien à l'identification de la meilleure commande au sens du critère (5).

La figure 6 donne les trajectoires correspondantes des courants. Les trois stratégies de contrôle optimal conduisent sensiblement aux mêmes trajectoires de courant, avec une légère différence au niveau de la fréquence. Par contre, la stratégie de commande vectorielle C.V.F.M. conduit à compenser un faible niveau de flux, imposé pour des raisons de rapidité de réponse, par une majoration de la composante $i_{\text {sq }}$ des courants (Fig. 7). 


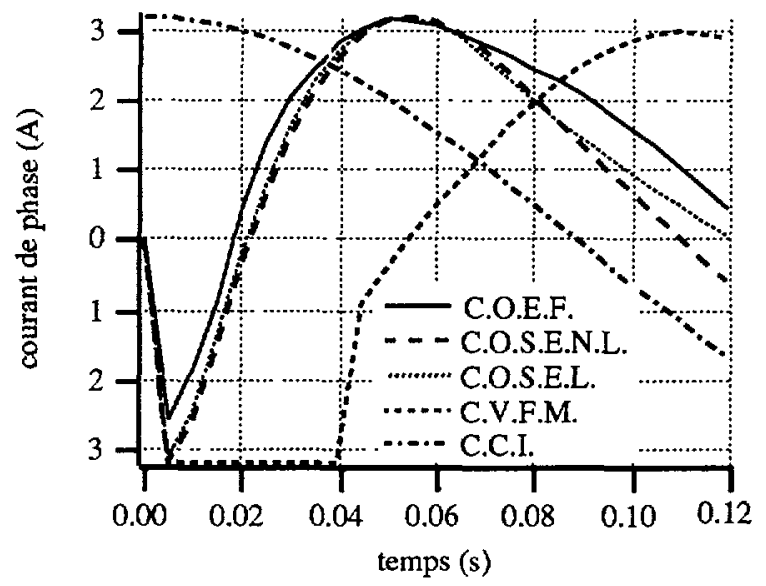

Fig. 6. - Modèle MCNL : amplitude des courants $\left(C^{*}=5 \mathrm{Nm}-I_{\operatorname{Max}}=2,25 \mathrm{~A}\right.$ eff $)$.

[MNCL model : command currents $\left(c^{*}=5 \mathrm{Nm}-I_{\mathrm{Max}}=2.25 \mathrm{~A}\right.$ eff $\left.).\right]$

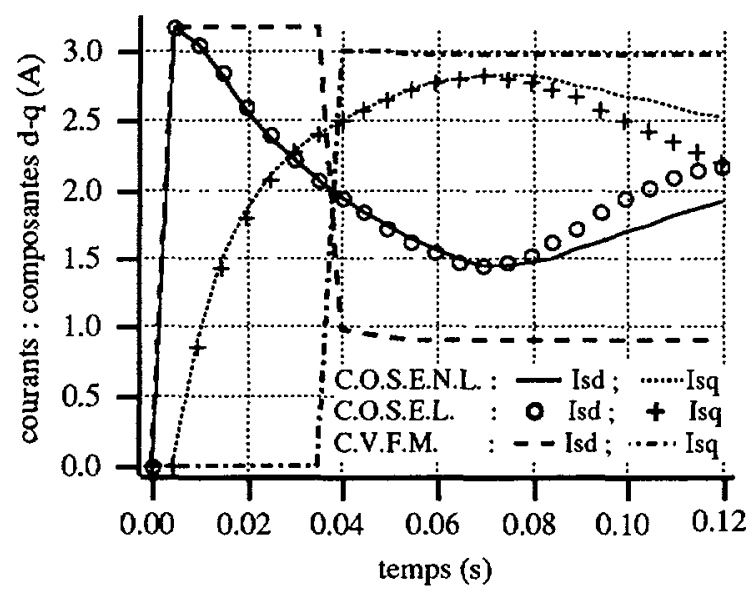

Fig. 7. - Modèle MCNL : composantes $d-q$ des courants ( $C^{*}=5 N m-I_{\text {Max }}=2,25$ A eff).

[MNCL model : $d-q$ components of the command currents $\left(c^{*}=5 N m-I_{\text {Max }}=2.25\right.$ A eff).]

6.4 CONFIGURATION MCNL : INFLUENCE DES CONTRAINTES SUR LES COURANTS. - D'après la figure 4, on constate que la réserve autorisée de courant n'est pas suffisante pour réaliser correctement la consigne de couple. Si on double la borne supérieure des courants $\left(I_{\text {Max }}=4,5 \mathrm{~A}\right.$ eff. $)$, avec la même consigne de couple de $5 \mathrm{Nm}$, on obtient les réponses en couple de la figure 8 .

La réserve de courant est maintenant suffisante pour réaliser rapidement la consigne de couple. Mais cette disponibilité conduit à saturer plus la machine. Pour juger dans quelles limites les stratégies de commande basées sur les modèles à constantes localisées sont aptes à tenir compte de situations très saturées, on utilise les séquences correspondantes de courant pour simuler le régime transitoire du moteur par un calcul éléments finis. La figure 9 reporte les réponses en couple correspondantes. Cette fois le modèle linéaire conduit à une réponse 


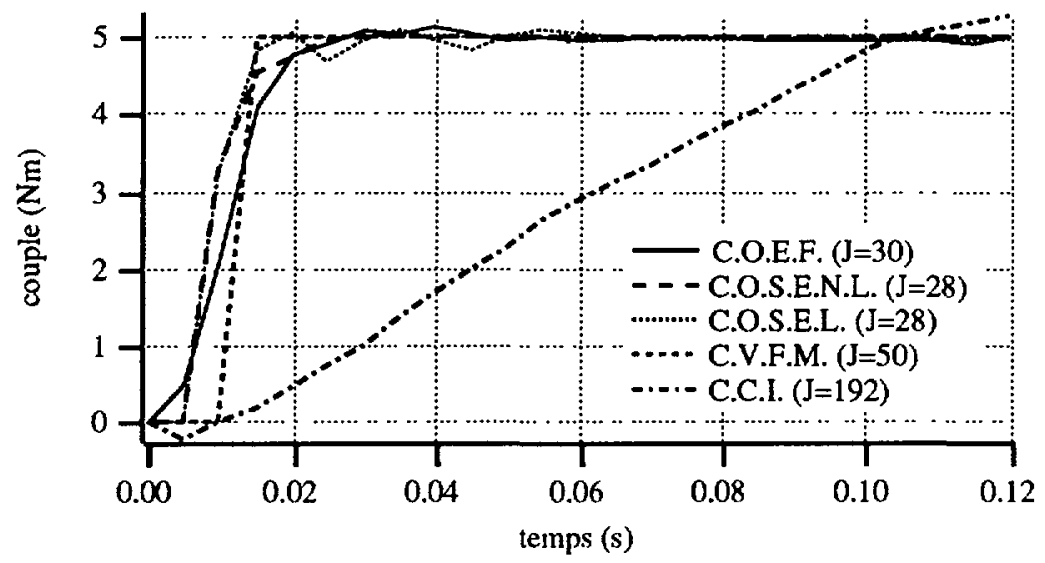

Fig. 8. - Modèle MCNL : réponses en couple $\left(c^{*}=5 \mathrm{Nm}-I_{\mathrm{Max}}=4,5 \mathrm{~A}\right.$ eff $)$.

[MCNL model $:$ torque responses $\left(c^{*}=5 \mathrm{Nm}-I_{\mathrm{Max}}=4.5 \mathrm{~A}\right.$ eff $\left.).\right]$

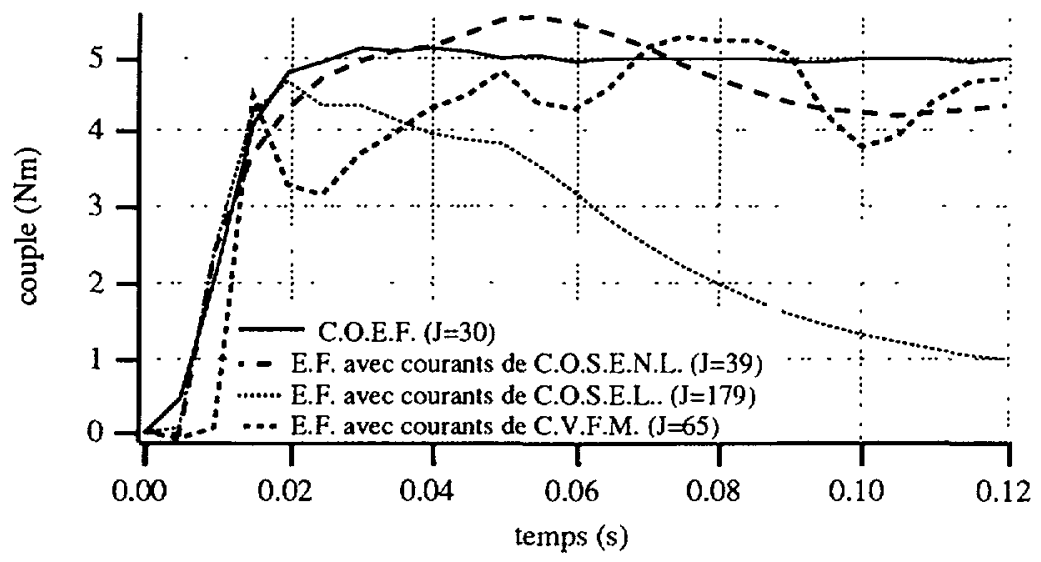

Fig. 9. - Modèle MCNL : simulation par éléments finis des réponses en couple réelles associées aux trajectoires de courant des modèles à constantes localisées $\left(c^{*}=5 \mathrm{Nm}-I_{\mathrm{Max}}=4,5 \mathrm{~A}\right.$ eff).

[MNCL model : torque responses with transient finite element field computation corresponding to current policies determined by optimal control with lumped-parameter model $\left(c^{*}=5 N m-I_{\operatorname{Max}}=4.5 \mathrm{~A}\right.$ eff $\left.).\right]$

tout à fait erronée, alors que les stratégies de commande C.O.S.E.N.L. et C.V.F.M. voient leurs performancem légèrement dégradées par rapport à celles de la stratégie C.O.E.F.

6.5 CONFIGURATION MARM : INFLUENCE DE LA SATURATION ET DES COURANTS INDUITS. - Les exemples précédents mettaient en ouvre un moteur à cage dont la constitution permettait de supposer que les paramètres variaient peu avec la fréquence. Afin d'illustrer la potentialité de la méthodologie proposée pour caractériser les modèles employés dans l'élaboration des commandes, on examine le cas extrême où le rotor à cage est remplacé par un rotor en acier massif. Dans ces conditions les caractéristiques du modèle à constantes 
localisées dépendent fortement du point de fonctionnement. Les simulations portent sur les stratégies C.O.E.F. et C.O.S.E.L. Les paramètres du modèle à constantes localisées de la stratégie C.O.S.E.L. sont ajustés au régime stationnaire donnant un couple de $3,4 \mathrm{mN}$ pour un courant de 2,25 A eff.

Les figures 10 et 11 reportent les réponses en couple et les trajectoires correspondantes des courants lorsque le moteur est initialement démagnétisé. La consigne de couple est de $3,4 \mathrm{Nm}$, et la contrainte sur les courants limite l'amplitude à 2,25 $\mathrm{A}$ efficace. Le modèle utilisé par la stratégie C.O.S.E.L. conduit à sous-estimer la fréquence des courants de commande. Ceci doit être relié à une variation trop importante des paramètres du moteur au cours du démarrage où le flux est initialement nul.

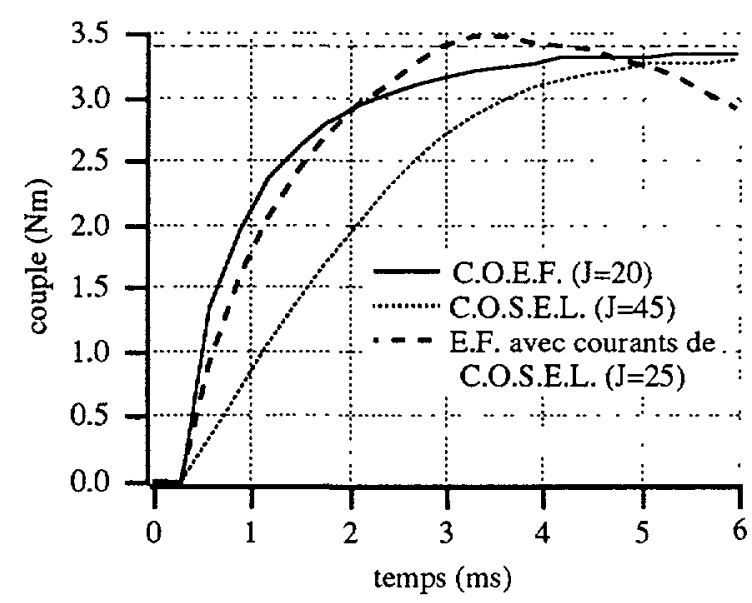

Fig. 10. - Modèle MARM : amplitude des courants $\left(c^{*}=3,4 \mathrm{Nm} \quad I_{\operatorname{Max}}=2,25\right.$ A eff $)$.

[MARM model : command currents $\left(c^{*}=3.4 \mathrm{Nm}-I_{\mathrm{Max}}=2.25 \mathrm{~A}\right.$ eff).]

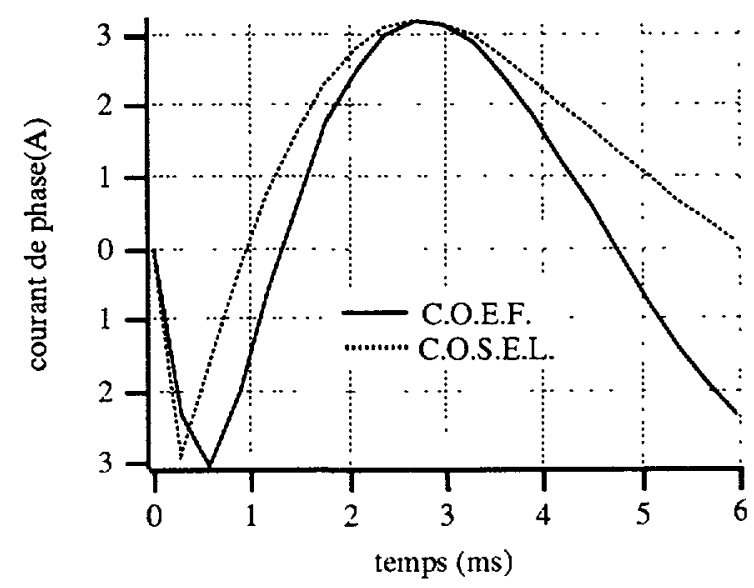

Fıg. 11. - Modèle MARM : composantes $d-q$ des courants.

[MARM model : $d-q$ components of the command currents.] 
L'inadaptation du modèle à constantes localisées est encore plus évidente quand la borne supérieure de l'amplitude des courants est doublée. C'est ce qu'indiquent les figures 12 et 13 où l'on a également reporté le couple obtenu avec la stratégie C.C.I. qui impose le courant

$$
i(t)=2,25 \sqrt{2} \cos (2 \pi \times 17 \times t) .
$$

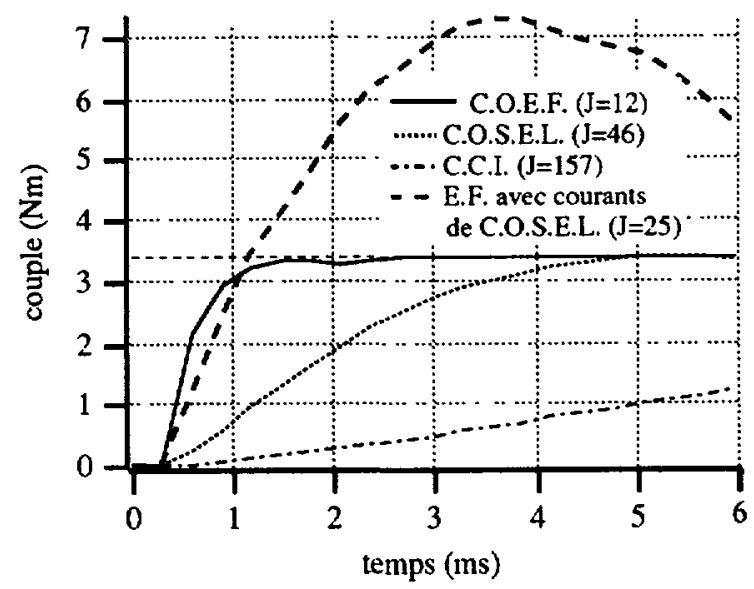

Fig. 12. - Modèle MARM : amplitude des courants $\left(c^{*}=3,4 N m-I_{\text {Max }}=4,5\right.$ A eff).

[MARM model : command currents $\left(c^{*}=3.4 N m-I_{\text {Max }}=4.5 \mathrm{~A}\right.$ eff $\left.).\right]$

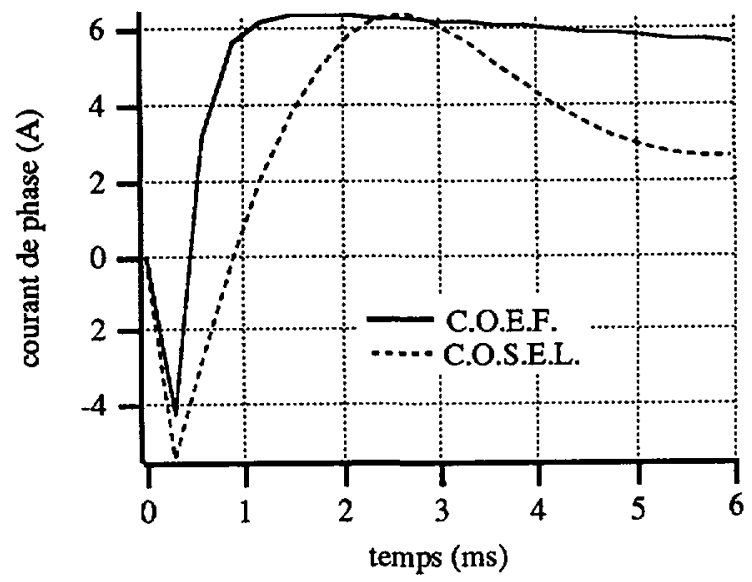

Fig. 13. - Modèle MARM : composantes $d-q$ des courants.

[MARM model : $d-q$ components of the command currents.]

La figure 14 donne la discrétisation retenue pour la modélisation éléments finis (240 éléments Q8 sur un pas polaire), ainsi que l'évolution de la pénétration du champ magnétique au rotor entre deux états intermédiaires sélectionnés le long de la trajectoire simposée par la stratégie C.O.E.F. 


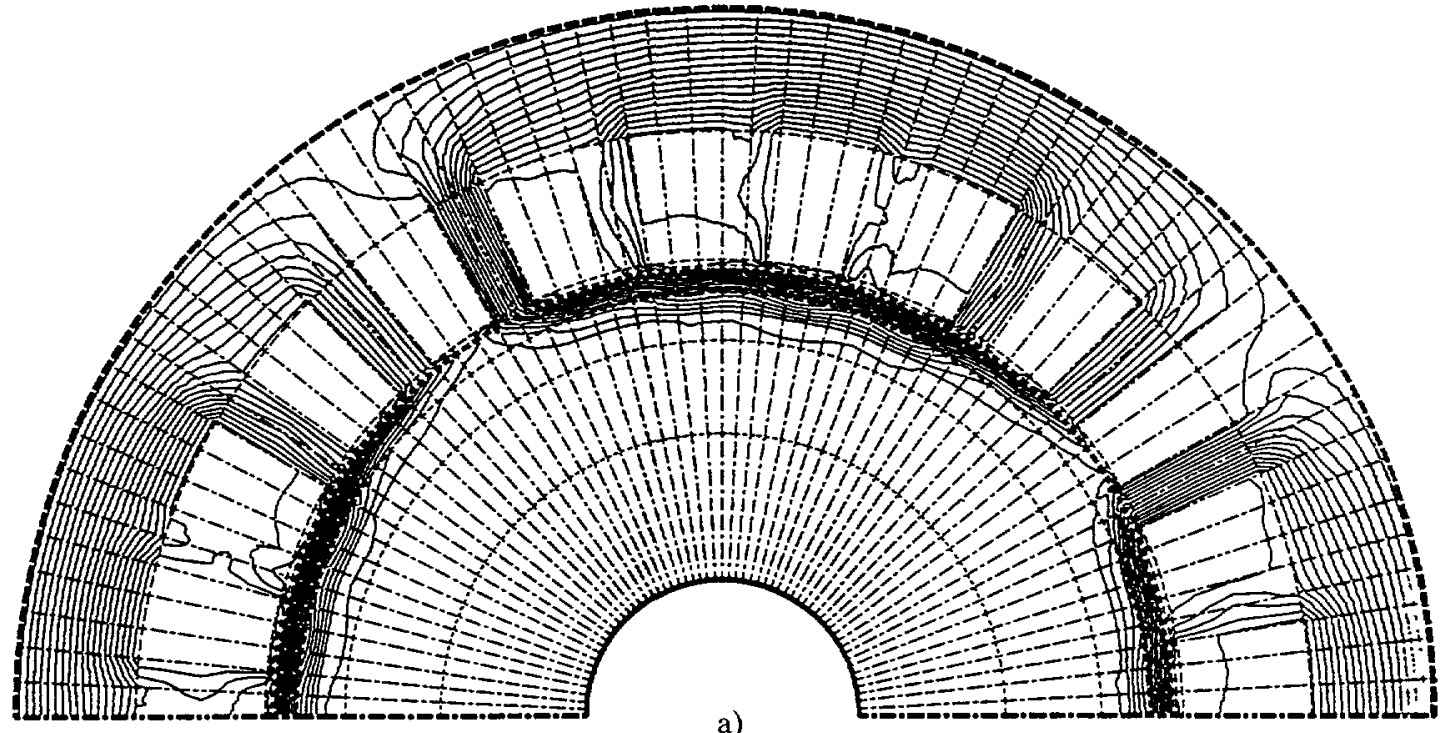

a)

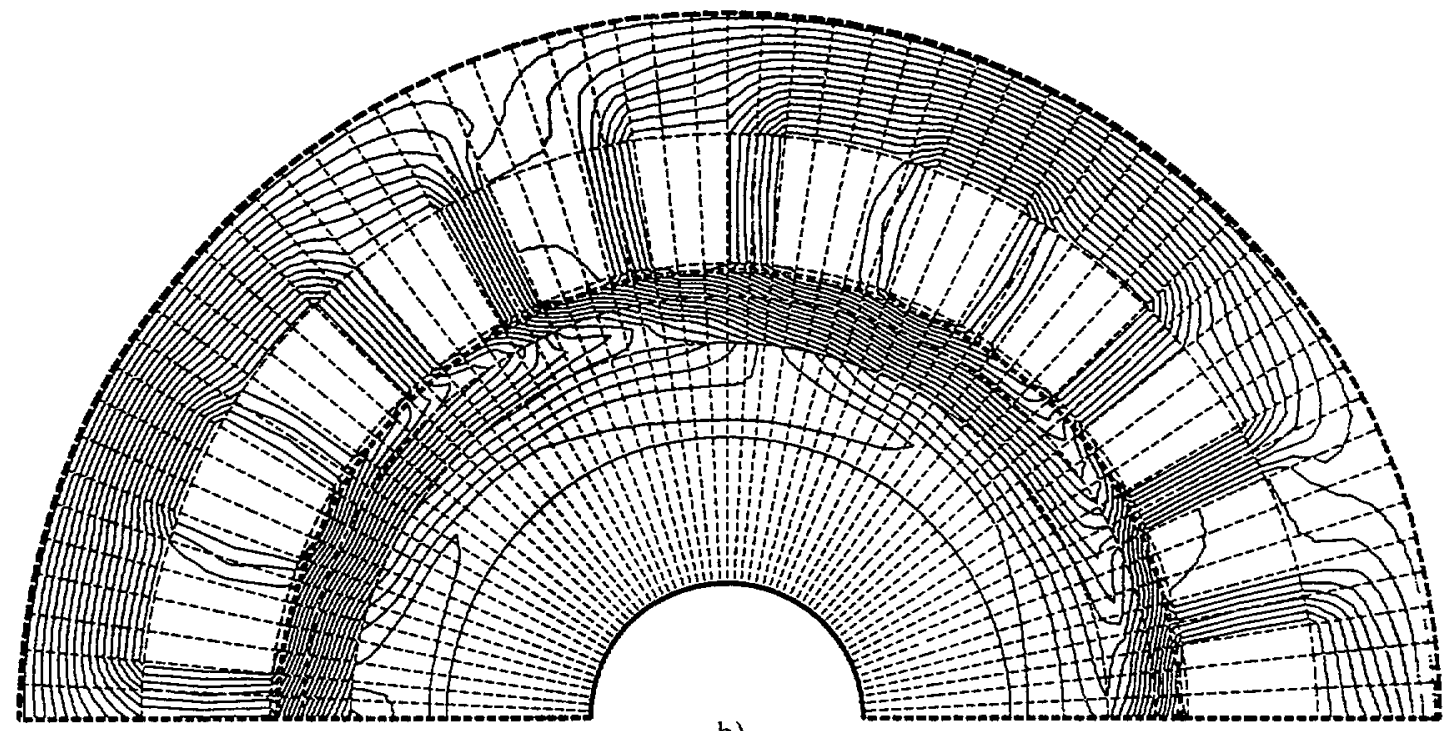

b)

Fig. 14. - Evolution du champ magnétique imposée par la stratégie C.O.E.F. a) $\Delta T=0,01$ s. b) $\Delta T=0,1 \mathrm{~s}$.

[Evolution of the flux distribution with C.O.E.F. strategy ; a) $\Delta T=0.01 \mathrm{~s}$, b) $\Delta T=0.1 \mathrm{~s}$.]

\section{Extension.}

La méthodologie présentée permet de résoudre le problème de la caractérisation de la capacité de réponse en couple d'un moteur. Il faut pour cela modifier le critère (15) comme

$$
J(\tilde{\mathbf{u}}) \leqslant J(\mathbf{u})=\int_{t_{0}}^{t_{\mathrm{f}}}-c_{\mathrm{cm}}(t) \mathrm{d} t \equiv-\int_{t_{0}}^{t_{\mathrm{f}}} \mathrm{X}(t)^{\mathrm{T}} \mathrm{QX}(t) \mathrm{d} t \quad \forall \mathbf{u} \in \mathrm{U}_{\mathrm{ad}}
$$


pour rechercher la commande qui maximise le couple. Les modifications correspondantes sont minimes, seule le deuxième membre de (22) est modifié.

A titre d'exemple recherchons la capacité limite en couple du modèle MCNL au démarrage avec la borne de courant à $4,5 \mathrm{~A}$ efficace. La figure 15 reporte cette réponse, ainsi que celle obtenue avec un courant d'amplitude et de fréquence constantes, soit

$$
i(t)=4,5 \sqrt{2} \cos (2 \pi \cdot 1,56 \cdot t),
$$

pour obtenir les $5 \mathrm{Nm}$ en régime stationnaire. La figure comporte également la réponse optimale obtenue avec le critère (15) caractéristique du suivi de trajectoire. On constate que les deux critères (15) et (43) conduisent, avec les mêmes contraintes sur la commande, à des réponses optimales en couple très différentes. La même remarque s'impose sur les stratégies correspondantes de commande en courant (Fig. 16); la stratégie qui maximise le couple conduit à une fréquence plus élevée. On observe également qu'une simple stratégie à courant

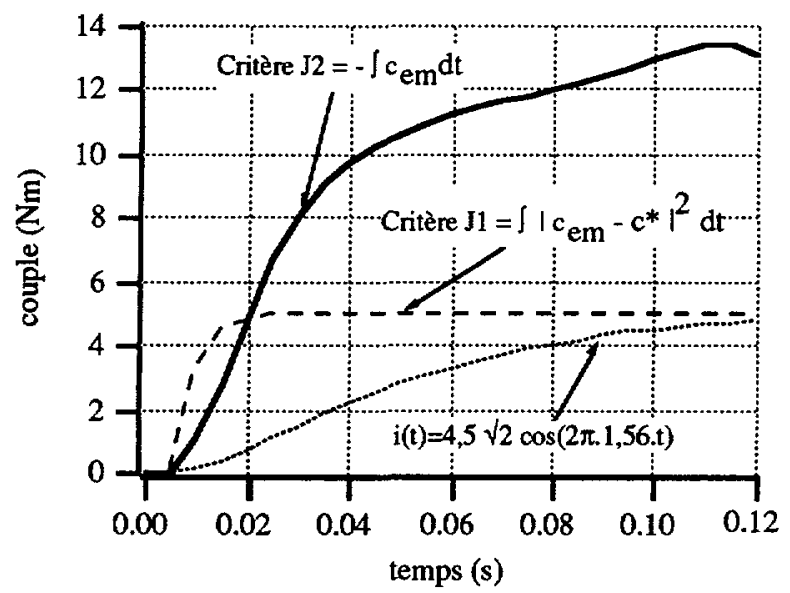

Fig. 15. - Modèle MCNL: capacité limite en couple.

[MCNL model : maximum torque response.]

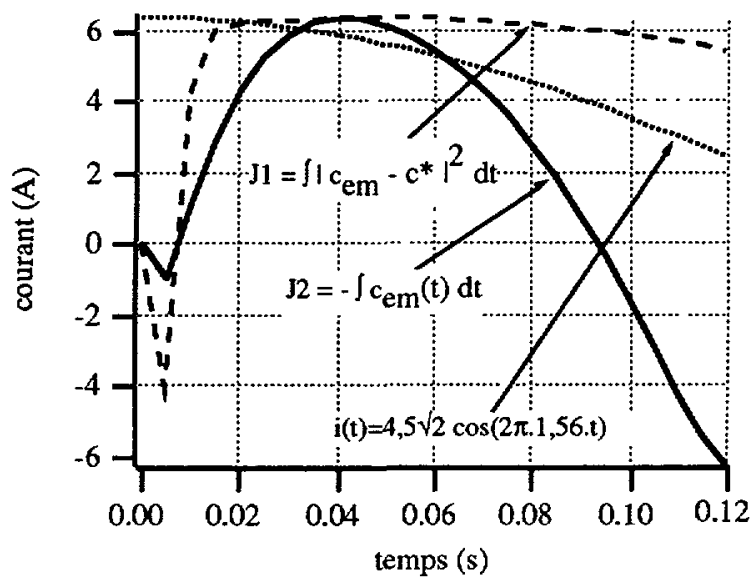

Fig. 16. - Modèle MCNL : courants $\left(I_{\text {Max }}=4,5\right.$ A eff).

[MCNL model : currents $\left(I_{\mathrm{Max}}=4.5 \mathrm{~A}\right.$ eff $\left.).\right]$ 
imposé peut conduire à très mal utiliser les potentialités de la machine si la fréquence est mal adaptée.

\section{Conclusion.}

On a proposé une méthodologie numérique générale d'identification des commandes optimales en couple des machines asynchrones. Sa principale caractéristique réside dans la modélisation de l'état électromagnétique de la machine par une formulation éléments finis, et d'un couplage avec des méthodes puissantes de programmation mathématique. Cette méthodologie permet de traiter des régimes dynamiques à flux variable en présence de saturation et de courants induits. La généralité de sa formulation autorise l'utilisation de différents critères d'optimisation. Elle s'avère un outil d'analyse intéressant pour juger de la qualité des modèles de machine utilisés par les commandes électroniques mises en œuvre. Sa principale limitation réside actuellement dans l'hypothèse de la vitesse constante. Une étude est en cours pour l'introduire comme variable d'état supplémentaire, ce qui permettra de traiter directement de la réponse optimale en temps de la mise en vitesse.

\section{Annexe 1}

\section{Paramètres du moteur.}

- moteur asynchrone ABB - série COMPAX-HEUX-80-L4

$-220 \mathrm{~V}-2,25 \mathrm{~A}, 50 \mathrm{~Hz}, 1500 \mathrm{t} / \mathrm{min}$

- résistance rotorique $R_{\mathrm{r}}=7,09 \Omega$

- inductance de fuites rotoriques $X_{\sigma \mathrm{r}}=0,056 \mathrm{H}$

- inductance de magnétisation :

$$
\begin{aligned}
& L_{\mathrm{m}}=0,667 \mathrm{H} \quad \text { pour } I_{\mathrm{M}} \leqslant 0,669 \mathrm{~A} \\
& =\frac{0,768}{0,481+I_{M}} \quad \text { pour } \quad 0,669 \mathrm{~A} \leqslant I_{M} \leqslant 1,999 \mathrm{~A} \\
& =\frac{1,471}{2.753+I_{M}} \quad \text { pour } \quad I_{M} \geqslant 1.999 \text {. }
\end{aligned}
$$

Caractéristiques de couple en régime stationnaire.

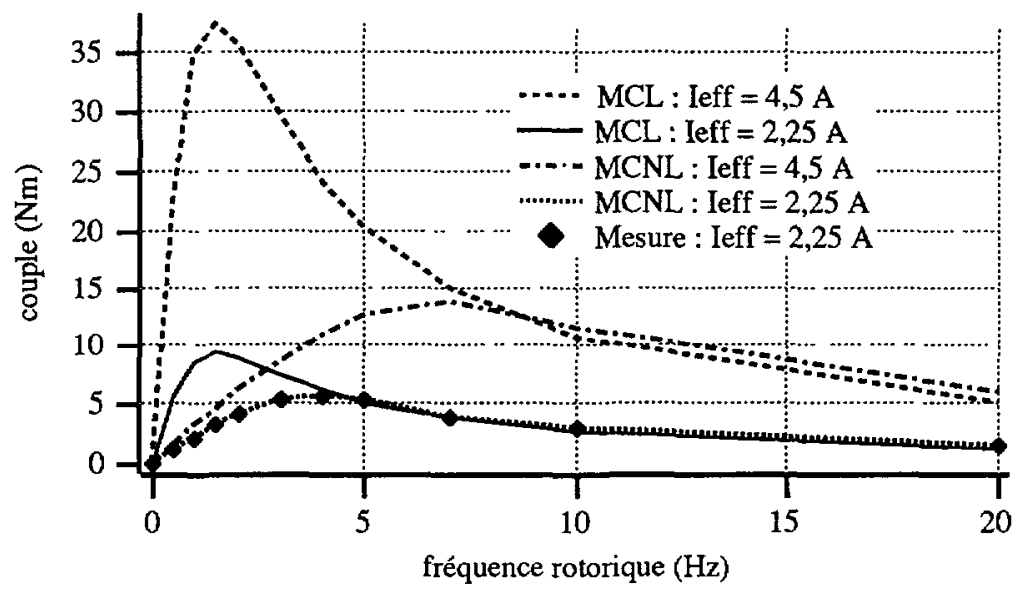




\section{Annexe 2}

Modèle à constantes localisées : équivalent de (10).

$$
\begin{gathered}
m \frac{\mathrm{d} \mathbf{x}(t)}{\mathrm{d} t}+k \mathbf{x}(t)=\mathbf{d}\left(\mathbf{i}_{\mathrm{s}}(\mathbf{u})\right)=b \mathbf{i}_{\mathrm{s}}(\mathbf{u}) \\
\mathbf{x}(t)=\left\{\psi_{\mathrm{rd}}, \psi_{\mathrm{rq}}, i_{\mathrm{rd}}, i_{\mathrm{rq}}\right\}^{\top}
\end{gathered}
$$

$\psi_{\mathrm{rd}}, \psi_{\mathrm{rq}}=$ composantes $\mathrm{d}-\mathrm{q}$ du flux rotorique $\psi_{\mathrm{r}}$ dans le référentiel statorique $i_{\mathrm{rd}}, i_{\mathrm{rq}}=$ composantes $\mathrm{d}-\mathrm{q}$ du courant rotorique $i_{\mathrm{r}}$ dans le référentiel statorique

$$
\begin{aligned}
& \mathbf{i}_{\mathrm{s}}(t)=\left\{I_{\mathrm{M}} \cos \psi, I_{\mathrm{M}} \sin \psi\right\}^{\top} \\
& \mathbf{u}(t)=\left\{I_{\mathrm{M}}(t), \psi(t)\right\}^{\top} \\
& m=\left[\begin{array}{llll}
1 & 0 & 0 & 0 \\
0 & 1 & 0 & 0 \\
0 & 0 & 0 & 0 \\
0 & 0 & 0 & 0
\end{array}\right], k=\left[\begin{array}{cccc}
\frac{1}{\tau_{\mathrm{r}}} & p \omega_{\mathrm{r}} & 0 & 0 \\
-p \omega_{\mathrm{r}} & \frac{1}{\tau_{\mathrm{r}}} & 0 & 0 \\
\frac{1}{L_{\mathrm{m}}+L_{\sigma \mathrm{r}}} & 0 & -1 & 0 \\
0 & \frac{1}{L_{\mathrm{m}}+L_{\sigma \mathrm{r}}} & 0 & -1
\end{array}\right] ; \\
& b=\left[\begin{array}{cc}
\frac{L_{\mathrm{m}}}{\tau_{\mathrm{r}}} & 0 \\
0 & \frac{L_{\mathrm{m}}}{\tau_{\mathrm{r}}} \\
\frac{L_{\mathrm{m}}}{L_{\mathrm{m}}+L_{\sigma \mathrm{r}}} & 0 \\
0 & \frac{L_{\mathrm{m}}}{L_{\mathrm{m}}+L_{\sigma \mathrm{r}}}
\end{array}\right] \\
& \tau_{\mathrm{r}}=\frac{L_{\mathrm{m}}+L_{\sigma \mathrm{r}}}{\mathrm{R}_{\mathrm{r}}}, \quad \omega_{\mathrm{r}}=\text { vitesse de rotation du rotor } \\
& c_{\mathrm{em}}=x^{\top} q x \text { avec: } q=\frac{p}{3}\left[\begin{array}{rrrr}
0 & 0 & 0 & -1 \\
0 & 0 & 1 & 0 \\
0 & 1 & 0 & 0 \\
-1 & 0 & 0 & 0
\end{array}\right]
\end{aligned}
$$

\section{Bibliographie}

[1] LeONHARD W., Control of electrical drives (Springer-Verlag, 1985).

[2] YAMAMURA S., AC Motors for High-performance applications (Marcel Dekker, INC, New York, 1986).

[3] LeVi E., Vuckovic V., Field-oriented control of induction machines in the presence of magnetic saturation, Elec. Mach. Pow. Sys. 16 (1989) 133-187.

[4] Fontaine J. C., Contribution à l'étude d'une commande en couple d'un actionneur asynchrone, Thèse de diplôme C.N.A.M. (1986). 
[5] Biedinger J. M., Poullain S., Yvon J. P., Optimal control of the torque of solid iron induction motor, Compumag Italie (juillet 1991).

[6] Sangwongxanich S., Ishida M., OKuma S., Iwata K., Manipulation of rotor flux for timeoptimal single-step velocity response of field-oriented induction machines, IEEE Trans. I. A. IA-24 (1988) 262-270.

[7] Lions J. L., Contrôle optimal de systèmes gouvernés par des équations aux dérivées partielles (Gauthier-Villars, 1968).

[8] Minoux M., Programmation mathématique: Théorie et algorithmes - Tome 2 (Dunod, 1983).

[9] Basile : un système de CAO pour l'automatisme, SIMULOG (France).

[10] Khater F., Lorenz R. D., Novotny D. W., Tang K., Selection of flux level in field-oriented induction machine controllers with consideration of magnetic saturation effects, IEEE Trans. I. A. IA-23 (1987) 276-282. 